Article

\title{
Short-Term Treatment with Alirocumab, Flow-Dependent Dilatation of the Brachial Artery and Use of Magnetic Resonance Diffusion Tensor Imaging to Evaluate Vascular Structure: An Exploratory Pilot Study
}

\author{
Thomas Metzner ${ }^{1,2} \mathbb{D}$, Deborah R. Leitner ${ }^{3} \mathbb{D}$, Gudrun Dimsity ${ }^{1}$, Felix Gunzer ${ }^{4}$, Peter Opriessnig ${ }^{4}$, \\ Karin Mellitzer ${ }^{3}$, Andrea Beck ${ }^{3}$, Harald Sourij ${ }^{3}$ D, Tatjana Stojakovic ${ }^{5}$, Hannes Deutschmann ${ }^{4}$, \\ Winfried März ${ }^{6,7,8}$, Ulf Landmesser ${ }^{9}$, Marianne Brodmann ${ }^{1}$, Gernot Reishofer ${ }^{4} \mathbb{D}$, Hubert Scharnagl ${ }^{8} \mathbb{D}$, \\ Hermann Toplak ${ }^{3, *(D)}$ and Günther Silbernagel ${ }^{1}$
}

check for updates

Citation: Metzner, T.; Leitner, D.R.; Dimsity, G.; Gunzer, F.; Opriessnig, P.; Mellitzer, K.; Beck, A.; Sourij, H.; Stojakovic, T.; Deutschmann, H.; et al. Short-Term Treatment with Alirocumab, Flow-Dependent Dilatation of the Brachial Artery and Use of Magnetic Resonance Diffusion Tensor Imaging to Evaluate Vascular Structure: An Exploratory Pilot Study. Biomedicines 2022, 10, 152. https://doi.org/ 10.3390/biomedicines10010152

Academic Editors: Alexander N. Orekhov and Igor A. Sobenin

Received: 30 November 2021

Accepted: 6 January 2022

Published: 11 January 2022

Publisher's Note: MDPI stays neutral with regard to jurisdictional claims in published maps and institutional affiliations.

Copyright: (C) 2022 by the authors. Licensee MDPI, Basel, Switzerland. This article is an open access article distributed under the terms and conditions of the Creative Commons Attribution (CC BY) license (https:// creativecommons.org/licenses/by/ $4.0 /)$.
1 Department of Internal Medicine, Division of Angiology, Medical University of Graz, 8036 Graz, Austria; metzner.thomas@gmx.at (T.M.); gudrun.dimsity@medunigraz.at (G.D.); marianne.brodmann@medunigraz.at (M.B.); guenther.silbernagel@medunigraz.at (G.S.)

2 Department of Medical Affairs, Sanofi-Aventis GmbH, 1100 Vienna, Austria

3 Department of Internal Medicine, Division of Endocrinology and Diabetology, Medical University of Graz, 8036 Graz, Austria; deborah.leitner@gmx.at (D.R.L.); karin.mellitzer@yahoo.de (K.M.);

Andrea.Beck@klinikum-graz.at (A.B.); ha.sourij@medunigraz.at (H.S.)

4 Department of Radiology, Clinical Division of Neuroradiology, Vascular and Interventional Radiology, Medical University of Graz, 8036 Graz, Austria; felix.gunzer@stud.medunigraz.at (F.G.); peter.opriessnig@alumni.tugraz.at (P.O.); hannes.deutschmann@medunigraz.at (H.D.); gernot.reishofer@medunigraz.at (G.R.)

5 Clinical Institute of Medical and Chemical Laboratory Diagnostics, University Hospital Graz, 8036 Graz, Austria; stojakovic@gmx.at

6 Department of Internal Medicine 5 (Nephrology, Hypertensiology, Endocrinology, Diabetology, Rheumatology), Mannheim Medical Faculty, University of Heidelberg, 68167 Mannheim, Germany; winfried.maerz@synlab.com

7 Synlab Academy, Synlab Holding Germany GmbH, 86156 Augsburg, Germany

8 Clinical Institute of Medical and Chemical Laboratory Diagnostics, Medical University of Graz, 8036 Graz, Austria; hubert.scharnagl@medunigraz.at

9 German Center for Cardiovascular Research (DZHK)-Partner Site Berlin, Department of Cardiology, Berlin Institute of Health, Charité University Medicine Berlin, 12200 Berlin, Germany; ulf.landmesser@charite.de

* Correspondence: hermann.toplak@medunigraz.at; Tel.: +43-316-385-80246 or +43-664-1045558 
detectable. More work would be needed to evaluate, whether fractional anisotropy may be useful in clinical atherosclerosis research.

Keywords: alirocumab; PCSK9; lipids; magnetic resonance; endothelial function; ultrasound

\section{Introduction}

Elevated Low-density lipoprotein (LDL) cholesterol is associated with increased atherosclerotic plaque volume [1]. On the other hand, LDL-cholesterol lowering is instrumental to reduce atherosclerotic plaque volume [2,3]. Treatment with proprotein convertase subtilisin/kexin type 9 (PCSK9) antibodies reduces plasma LDL-cholesterol by as much as $60 \%$ [4-6]. In addition, treatment with PCSK9-antibodies has been demonstrated to be associated with plaque regression and higher plaque stability measured by intravascular ultrasound and optical coherence tomography [7-10]. However, intravascular ultrasound and optical coherence tomography both carry the risk of invasive procedures such as hematoma after an arterial puncture, infection, allergy against contrast media, or catheter-induced endothelial damage. Carotid intima-media thickness is commonly used for the non-invasive estimation of plaque formation [11,12]. This method is safe but imprecise. Brachial artery flow-dependent dilatation represents another ultrasound-based method that is considered a measure of the atherosclerotic process. However, it does not provide information on structural changes of the arteries [13,14]. Reliable non-invasive procedures to measure atherosclerotic changes are therefore needed [15].

We have introduced a completely novel method based on magnetic resonance diffusion tensor imaging to quantify structural changes of the carotid arteries. Ex-vivo, this method was demonstrated to have a high spatial resolution in visualizing collagen fiber bundles of the atherosclerotic fibrous cap [16]. In addition, this method appeared to provide information on vascular aging in a cohort of healthy males considering the inverse relationship between age and fractional anisotropy. This diffusion tensor imaging-based parameter is considered to reflect the proper alignment of collagen fiber bundles of the carotid arteries [17].

The present study was designed to explore the usability of this novel non-invasive magnetic resonance imaging method in patients with atherosclerotic vascular disease and to collect longitudinal clinical data on fractional anisotropy for the first time. Moreover, the objective was to investigate the effects of short-term treatment with alirocumab on brachial artery flow-dependent dilatation as well as inflammatory and vascular biomarkers.

\section{Methods}

\subsection{Study Design}

The ALIROCKS study (NCT03559309) is a prospective, monocentric, and longitudinal pilot study with 24 patients scheduled for treatment with alirocumab. Treatment with alirocumab was initiated based on the current guidelines for the treatment of dyslipidemia of the European Atherosclerosis Society and the European Society of Cardiology [18]. Inclusion criteria were atherosclerotic cardiovascular disease and elevated LDL-cholesterol despite maximum tolerated oral lipid-lowering therapy. Exclusion criteria were age $<18$ years, pregnancy, previous treatment with PCSK9-antibodies, and known contraindication for magnetic resonance imaging of the carotid artery (e.g., claustrophobia). There were 2 visits to the study site, the first before initiation of treatment with alirocumab and the second after 10 weeks of treatment. Diagnostic procedures of the first visit included a physical examination and a questionnaire on the medical history. Laboratory testing, ultrasound-based vascular imaging, and 2-dimensional cardiovascular magnetic resonance imaging of the carotid arteries were performed at the first and second visits.

The primary outcome measure was defined as the change of mean carotid vessel wall fractional anisotropy. Secondary outcome measures included the change of flow-dependent 
dilatation of the brachial artery, change of carotid intima-media thickness, and change of systemic inflammation in response to treatment with alirocumab. The clinical trial was conducted in accordance with the Declaration of Helsinki and was approved by the Austrian Federal Office for Safety in Health Care (EudraCT: 2018-000981-12) and the Ethics Committee of the Medical University of Graz (protocol number: 29-519 ex 16/17, approval date: 6 April 2018). All participants gave written informed consent.

\subsection{Clinical Characterization}

At baseline, demographics, and clinical characteristics such as age, sex, smoking habits, concomitant diseases, and lipid medication were documented. Familial hypercholesterolemia was reported according to medical history. Patients not receiving high-intensity statins defined as $\geq 40 \mathrm{mg}$ atorvastatin or $\geq 20 \mathrm{mg}$ rosuvastatin at baseline were categorized as having partial or complete statin intolerance.

\subsection{Cardiovascular Magnetic Resonance Imaging}

High-resolution diffusion tensor imaging was performed with a $2 \mathrm{D}$ diffusion acquisition scheme. A readout-segmented Echo-Planar-Imaging (rs-EPI MRI) sequence was used on a 3T whole-body MR scanner (Prisma fit, Siemens Medical Solutions, Erlangen, Germany) with a $2 \times 4$ channel multifunctional coil (NORAS MRI products GmbH, Höchberg, Germany) and FOV $=189 \times 189 \mathrm{~mm}^{2}$, matrix $=346 \times 346$, slice thickness $=10 \mathrm{~mm}$, $\mathrm{TR}=2 \mathrm{RR}$ intervals, trigger delay $=50 \mathrm{~ms}$ to acquire image in the diastolic phase, $\mathrm{TE}=93 \mathrm{~ms}$, GRAPPA $=2$, number of readout segments $=9$, b-values $=0,200,400,600 \mathrm{~s} / \mathrm{mm}^{2}$, acquisition time $=\sim 12$ min. 18 gradient directions were defined on a hemicycle within a plane oriented perpendicular to the longitudinal axis of the carotid artery. Fractional anisotropy is a scalar value without a unit between one and zero which describes the degree of anisotropy of a diffusion process (Figure S1). A value of one means that the process of diffusion is directed along one axis and is fully restricted along with all other directions, while a value of zero means that diffusion is isotropic or unrestricted in all directions. Lower values of fractional anisotropy are considered to reflect pathological changes of the carotid vessel wall structure $[16,17]$. The semiautomatic evaluation of fractional anisotropy was not conducted in a blinded design per study protocol. However, the person responsible for image analysis was not informed about the patient numbers and the time points of the measurements (baseline or week 10).

\subsection{Laboratory Procedures}

Blood samples were taken in the fasting state. LDL-cholesterol and HDL-cholesterol were assessed by lipoprotein-electrophoresis, cholesterol and triglycerides were measured by photometry. Apolipoproteins (Apo) and C-reactive protein (CRP) were measured with immunoturbidimetry, and interleukin-6 (IL-6) was measured with an electrochemiluminescence immunoassay. Monocyte chemoattractant protein-1 (MCP-1, by TECAN, Männedorf, Switzerland, ID: 30150435), Vascular endothelial growth factor (VEGF, by IBL, Minneapolis, MN, USA, ID: JP27171), and P-Selectin/CD62P (by Bio-Techne, Minneapolis, MN, USA, ID: DPSE00), were measured by enzyme-linked immunosorbent assays.

\subsection{Intima-Media Thickness and Flow-Dependent Dilatation}

All vascular ultrasound measurements were performed by a single experienced technician using a linear array transducer, 8-13 MHz (Sequoia 512, ACUSON Corp., Charleston Rd., Mountain View, CA, USA). Carotid intima-media thickness was defined as the mean of the left and right intima-media thickness which was each based on three different measurements in a one-centimeter-long segment of the common carotid artery. For the standardized measurement of flow-dependent dilatation of the brachial artery, a blood pressure cuff on the forearm was inflated for $5 \mathrm{~min}$ with a constant pressure of $50 \mathrm{mmHg}$ above the systolic brachial artery blood pressure. The post-ischemic brachial arterial diameter was measured 
$45 \mathrm{~s}$ after cuff release. Flow-dependent dilatation was defined as the percent change of the brachial artery diameter in response to ischemia compared to the baseline diameter $[13,14]$.

\subsection{Statistical Analysis}

The statistical design of this pilot trial was explorative and descriptive. The study duration and sample size were based on previous studies investigating the effects of shortterm lipid-lowering on endothelial function [19-24]. Baseline characteristics are specified as numbers (percentages) in cases of categorical variables and as means (standard deviations) in cases of continuous variables. Changes in between baseline and week 10 were tested with the paired samples $t$-test and/or related samples Wilcoxon signed-rank test when Shapiro-Wilk analysis suggested non-normal distribution. The SPSS 26.0 statistical program was used (SPSS Inc., Chicago, IL, USA).

\subsection{Monitoring}

Source data verification was performed for carotid vessel wall fractional anisotropy assessed by cardiovascular magnetic resonance and for flow-dependent dilatation of the brachial artery measured by ultrasound. In addition, informed consent forms were monitored. The monitoring was conducted by an independent, external clinical research organization (MM Clinical Services \& Consulting GmbH, Hart bei Graz, Austria).

\section{Results}

\subsection{Study Population}

The recruitment phase for the 24 participants lasted from 20 June 2018 to 30 January 2020. About $80 \%$ of the participants received $75 \mathrm{mg}$ of alirocumab every two weeks and $20 \%$ received $150 \mathrm{mg}$ alirocumab every two weeks. Two patients only injected alirocumab twice, at baseline and week 2. The reasons for their non-adherence were documented as drug access and communication barriers in the clinical routine. Four patients had minor changes in their oral lipid-lowering therapies during the trial. Among these, two patients stopped their red yeast rice product, one stopped ezetimibe, and another one reduced the oral combination therapy from $80 \mathrm{mg}$ atorvastatin plus $10 \mathrm{mg}$ ezetimibe to $20 \mathrm{mg}$ atorvastatin monotherapy. Due to the COVID-19 pandemic and the subsequent implementation of national countermeasures restricting all hospital visits to essential interventions only, the clinical trial had to be terminated before the last 3 participants had completed the study. Hence, 19 patients completed the trial according to the protocol (Table 1). The mean ( \pm standard deviation) treatment duration with alirocumab for participants who had completed the trial was $10.0( \pm 0.7)$ weeks.

Table 1. Study Population and Alirocumab Dosage.

\begin{tabular}{cccc}
\hline Definition & Population (N) & $\begin{array}{c}\text { Alirocumab } 75 \mathbf{~ m g} \\
\mathbf{( \% )}\end{array}$ & $\begin{array}{c}\text { Alirocumab 150 mg } \\
\mathbf{( \% )}\end{array}$ \\
\hline Recruitment & 24 & $19(79.2)$ & $5(20.8)$ \\
Completion & 19 & $16(84.2)$ & $3(15.8)$ \\
\hline
\end{tabular}

Legend: Trial completion population $(n=19)$ includes patients attending their baseline plus week 10 visit and injected alirocumab as planned. Three patients could not conduct their scheduled week 10 visit due to COVID-19 countermeasures. Two patients injected alirocumab only twice at baseline and at week 2 but not beyond.

\subsection{Characteristics of Trial Participants}

The cohort included middle-aged and elderly females and males. All 19 participants had documented coronary heart disease. Among these, 7 patients had poly-vascular disease with an additional diagnosis of cerebral and/or peripheral artery occlusive disease. The vast majority, 16 participants, had received the coronary intervention or bypass surgery. All 3 patients without prior coronary intervention or surgery had poly-vascular disease with coronary stenosis $(50 \%)$ confirmed by cardiac computed tomography and carotid artery occlusive disease. One of the 3 patients without prior coronary intervention or surgery 
also had peripheral artery disease. Familial hypercholesterolemia was documented in 2 patients. A total of 16 study participants had partial or complete statin intolerance. Hence, very few participants received high-intensity statins at baseline. However, the proportion of patients taking ezetimibe as concomitant medication was high (Table 2 and Table S1).

Table 2. Characteristics of Trial Participants (Trial Completion).

\begin{tabular}{|c|c|}
\hline Characteristic & $\begin{array}{l}\text { Alirocumab } \\
\quad(N=19)\end{array}$ \\
\hline Age-yr & $66(9)$ \\
\hline Female sex-n (\%) & $9(47.4)$ \\
\hline Male sex-n (\%) & $10(52.6)$ \\
\hline Smoker ${ }^{\mathrm{a}}-\mathrm{n}(\%)$ & $9(47.4)$ \\
\hline Current Smoker ${ }^{\mathrm{b}}-\mathrm{n}(\%)$ & $4(21.1)$ \\
\hline \multicolumn{2}{|l|}{ Concomitant Diseases-n (\%) } \\
\hline Cardiovascular Disease & $19(100)$ \\
\hline Coronary Heart Disease & $19(100)$ \\
\hline Coronary Intervention or Surgery & $16(84.2)$ \\
\hline Documentation of Coronary Stenosis ${ }^{c}$ & $3(15.8)$ \\
\hline Peripheral Artery Disease & $3(15.8)$ \\
\hline Cerebral Artery Disease & $6(31.6)$ \\
\hline Chronic Kidney Disease & $4(21.1)$ \\
\hline Familial Hypercholesterolaemia d & $2(10.5)$ \\
\hline Adiposity & $4(21.1)$ \\
\hline Type-2 Diabetes Mellitus & $4(21.1)$ \\
\hline Type-1 Diabetes Mellitus & $0(0)$ \\
\hline Hypertension & $15(78.9)$ \\
\hline \multicolumn{2}{|l|}{ Number of prior Cardiovascular Events ${ }^{\mathrm{e}}-\mathrm{n}(\%)$} \\
\hline Three & $2(10.5)$ \\
\hline Two & $5(26.3)$ \\
\hline One & $9(47.4)$ \\
\hline Zero & $3(15.8)$ \\
\hline \multicolumn{2}{|l|}{ Concomitant Lipid Medication-n (\%) } \\
\hline High-Intensity Statins $\mathrm{f}$ & $3(15.8)$ \\
\hline Statins & $5(26.3)$ \\
\hline Ezetimibe & $13(68.4)$ \\
\hline Dietary Supplements $g$ & $5(26.3)$ \\
\hline Statin Intolerance ${ }^{\mathrm{h}}$ & $16(84.2)$ \\
\hline
\end{tabular}

Legend: Values are numbers (percentages) or means (standard deviations) for categorical and continuous variables, respectively. ${ }^{a}$ Current or former smoker. ${ }^{b}$ Documented as a current smoker, or no stop date documented. ${ }^{c}$ Confirmed by cardiac computed tomography but without documentation of prior cardiovascular event (e.g., stroke, myocardial infarction, or percutaneous intervention). ${ }^{\mathrm{d}}$ According to medical records. ${ }^{\mathrm{e}}$ Documented as a stent, balloon, coronary artery bypass graft, myocardial infarction, or percutaneous intervention, prior strokes/transient ischemic attacks. ${ }^{\mathrm{f}}$ Documented as $\geq 40 \mathrm{mg}$ of atorvastatin or $\geq 20 \mathrm{mg}$ of rosuvastatin. ${ }^{\mathrm{g}}$ Exclusively red yeast rice combination products (monacolin $\mathrm{K}) .{ }^{\mathrm{h}}$ Patients that did not receive high-intensity statins at baseline (includes partial or complete intolerance).

\subsection{Effects of Alirocumab on Lipids, Systemic Inflammation, and Vascular Biomarkers}

Mean LDL-cholesterol was reduced by $76 \mathrm{mg} / \mathrm{dL}$ in response to treatment with alirocumab (relative reduction of 49\%; Table 3 and Figure S2 of Supplementary Materials). Apo-B was reduced by $39 \%$, cholesterol by $32 \%$, and $\mathrm{Lp}(\mathrm{a})$ by $16 \%$. Two patients had documented familial hypercholesterolemia and in one of them, baseline LDL-cholesterol was $>400 \mathrm{mg} / \mathrm{dL}$. Eight participants had Lp(a) values $>50 \mathrm{mg} / \mathrm{dL}$. In this subgroup Lp(a) was reduced from $94 \mathrm{mg} / \mathrm{dL}$ to $81 \mathrm{mg} / \mathrm{dL}$ resulting in a mean absolute reduction of 14 (12) $\mathrm{mg} / \mathrm{dL}(p=0.018)$. We did not detect changes in the systemic inflammatory biomarkers CRP and MCP-1 in response to treatment with alirocumab. There was only a minor but statistically significant reduction of IL-6. The vascular biomarkers P-selectin/CD62P and VEGF did not change in response to alirocumab treatment (Table 3). 
Table 3. Effects of Alirocumab on Lipids, Systemic Inflammation, and Vascular Biomarkers.

\begin{tabular}{|c|c|c|c|c|c|c|c|c|}
\hline Parameters & Baseline & $\pm \mathbf{S D}$ & Week 10 & $\pm \mathbf{S D}$ & $\begin{array}{l}\text { Absolute } \\
\text { Change }\end{array}$ & \pm SD & $\begin{array}{c}\text { Relative } \\
\text { Change (\%) }\end{array}$ & $p$-Value \\
\hline \multicolumn{9}{|l|}{ Standard Lipids ${ }^{a}$} \\
\hline LDL-Cholesterol (mg/dL) & 154 & 85 & 78 & 91 & -76 & 44 & -49 & $<0.001$ \\
\hline Apo-B (mg/dL) & 124 & 56 & 76 & 65 & -48 & 28 & -39 & $<0.001$ \\
\hline Cholesterol (mg/dL) & 244 & 104 & 166 & 102 & -78 & 41 & -32 & $<0.001$ \\
\hline $\mathrm{Lp}(\mathrm{a})(\mathrm{mg} / \mathrm{dL})$ & 51 & 43 & 43 & 39 & -8 & 11 & -16 & 0.007 \\
\hline Triglycerides (mg/dL) & 148 & 87 & 132 & 65 & -17 & 73 & -11 & 0.332 \\
\hline HDL-Cholesterol (mg/dL) & 50 & 13 & 59 & 16 & +9 & 15 & +18 & 0.018 \\
\hline \multicolumn{9}{|l|}{ Systemic Inflammation ${ }^{b}$} \\
\hline $\mathrm{CRP}(\mathrm{mg} / \mathrm{L})$ & 2.7 & 3.5 & 2.6 & 3.1 & -0.1 & 1.7 & -4 & 0.902 \\
\hline IL-6 (pg/mL) & 3.1 & 1.7 & 2.7 & 1.4 & -0.5 & 0.9 & -13 & 0.030 \\
\hline $\mathrm{MCP}-1(\mathrm{pg} / \mathrm{mL})$ & 617 & 219 & 623 & 188 & +6.2 & 99 & +1 & 0.789 \\
\hline \multicolumn{9}{|l|}{ Vascular Biomarkers ${ }^{c}$} \\
\hline VEGF $(\mathrm{pg} / \mathrm{dL})$ & 6.2 & 6.1 & 7.5 & 7.8 & +1.3 & 5.7 & +21 & 0.409 \\
\hline P-Selectin/CD62P (ng/mL) & 35.4 & 8.0 & 35.5 & 10.2 & +0.1 & 7.4 & +0.3 & 0.963 \\
\hline
\end{tabular}

Legend: Table shows mean values and changes; Trial-completion analysis $(\mathrm{n}=19)$; Paired $t$-test with a two-sided $p$-value. ${ }^{\text {a }}$ LDL-Cholesterol and HDL-Cholesterol were assessed by lipid-electrophoresis; triglycerides and total cholesterol were measured by photometry; lipoproteins were determined by immunoturbidimetry. Units are $\mathrm{mg} / \mathrm{dL} .{ }^{\mathrm{b}} \mathrm{CRP}$ was measured with immunoturbidimetry; IL- 6 was measured with an electrochemiluminescence immunoassay; MCP-1 was measured by enzyme-linked immunosorbent assay. ${ }^{\mathrm{c}}$ Parameters were measured by enzyme-linked immunosorbent assays. VEGF: Five patients had values below the limit of detection $(\mathrm{n}=14)$.

\subsection{Effects of Alirocumab on Vascular Structure and Function}

The structural investigation of the carotid arteria using magnetic resonance and standard ultrasound-based imaging showed no changes in response to 10 weeks of treatment with alirocumab (Figures 1 and 2). The endothelial function of the arteria brachialis also did not significantly change $(+41 \%, p=0.241)$. There was a trend towards an inverse relationship between LDL-cholesterol reduction and the change of flow-dependent dilatation ( $r=0.402, p=0.098$; Figure S3 of Supplementary Materials). We obtained valid fractional anisotropy measurements for baseline and week 10 for 13 patients and valid flowdependent dilatation and carotid intima-media thickness measurements for 18 patients. The considerable drop-out number for the magnetic resonance imaging resulted from one patient's anatomy (adiposity with short neck), two patients with claustrophobia, and an outlier value that was excluded for analysis. The remaining two patients missed their scheduled visits. One measurement was missing for both ultrasound-based techniques.

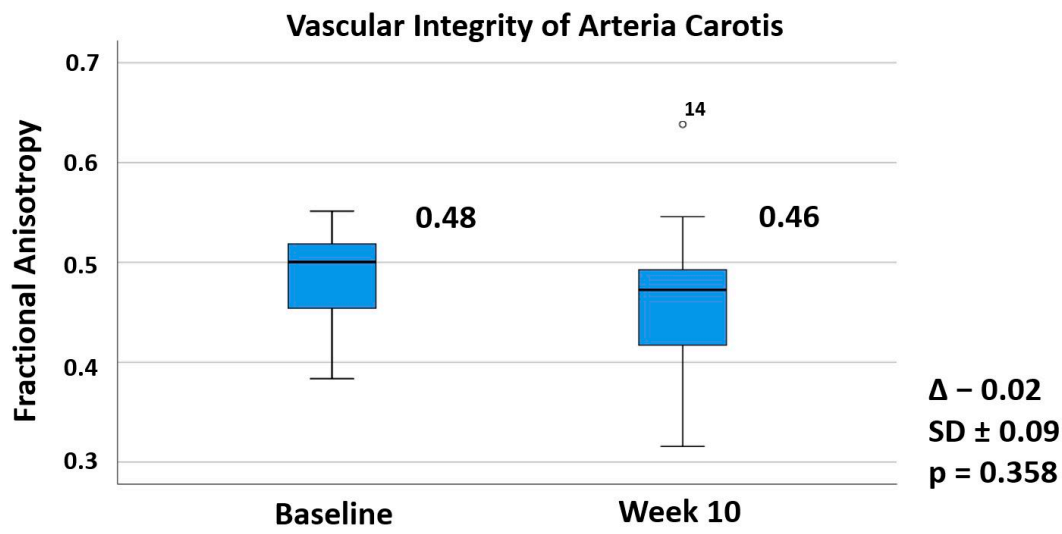

Figure 1. Effects of Alirocumab on a Novel Magnetic Resonance-based Vascular Parameter. Legend: Assessment of vascular integrity by evaluating fractional anisotropy of the carotid vessel wall. The numerical data shows mean values at baseline and week 10 of alirocumab treatment with mean change, standard deviation, and $p$-value, while boxplots graphically represent medians and interquartiles. The small circle with the number 14 at the right upper side of the figure identifies an outlier measurement of patient number 14 at week 10. Outliers are defined as values between $1.5 \times$ and $3 \times$ interquartile ranges from the end of a box. Fractional anisotropy is a scalar value without a unit between one and zero. Lower values of fractional anisotropy are considered to reflect pathological changes of the carotid vessel wall structure. Trial-completion analysis of patients with two valid assessments for statistical analysis $(n=13)$. Paired $t$-test with a two-sided $p$-value. 
A.

Endothelial Function of Arteria Brachialis
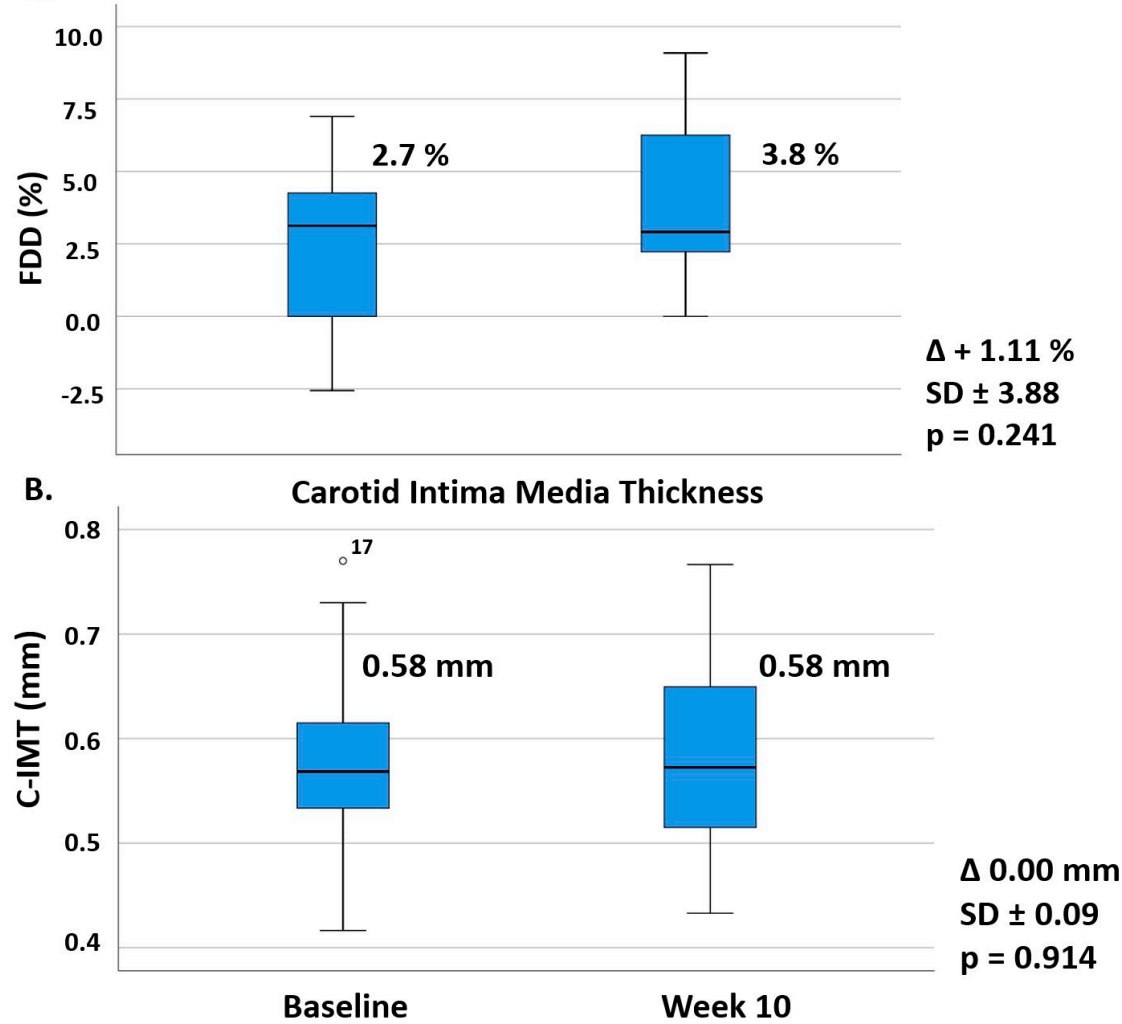

Figure 2. Effects of Alirocumab on Established Ultrasound-based Vascular Parameters. Legend: The numerical data shows mean values at baseline and after 10 weeks of alirocumab treatment with mean change, standard deviation, and $p$-value, while boxplots graphically represent medians and interquartiles. The small circle with the number 17 at the left upper side of the figure identifies an outlier measurement of patient number 17 at baseline. Outliers are defined as values between $1.5 \times$ and $3 \times$ interquartile ranges from the end of a box. Trial-completion analysis with two valid assessments for statistical analysis $(\mathrm{n}=18)$; Paired $t$-test with a two-sided $p$-value. (A) Flow-dependent dilatation (FDD) of the arteria brachialis. (B) Carotid intima-media thickness (C-IMT) of the vessel wall.

\section{Discussion}

Fractional anisotropy is a completely novel magnetic resonance imaging-based parameter measuring the structural integrity of the vasculature. Due to anatomic issues (adiposity with short neck), claustrophobia, an outlier value, and the time-consuming measurement regimen, values were only obtained for a subgroup of the ALIROCKS study limiting the current clinical and scientific applicability of the method. During the ALIROCKS study, factional anisotropy did not significantly change, as expected. The short duration of treatment with alirocumab, which resulted in a 49\% LDL-cholesterol reduction, and the low sample did not suggest significant changes in vascular structure. Moreover, there are no established cut-off values differentiating normal fractional anisotropy values from pathologic ones. The reproducibility of this magnetic resonance imaging method was analyzed in a previous study of our group in healthy subjects by providing the coefficients of variation. Four repeated measurements in four healthy male volunteers showed coefficients of variation between $2.5 \%$ and $5.4 \%$. This previous cross-sectional evaluation in healthy subjects also demonstrated an inverse relationship between age and fractional anisotropy. These participants had fractional anisotropy ranging from around 0.70 at 27 years to 0.56 at 57 years of age [17]. The present population had a mean age of 66 years and a mean baseline fractional anisotropy of 0.48 , which is consistent with the concept of a progressive decrease of fractional anisotropy with increasing age. Associations of fractional anisotropy with LDL cholesterol were not detectable in the present small cohort. 
There is a scarce of non-invasive methods to reliably measure atherosclerosis. Using invasive methods such as optical coherence tomography, PCSK9-antibodies have been suggested to stabilize the fibrous cap of coronary atherosclerotic lesions and to reduce the size of lipid-rich plaques. Two single-center trials used optical coherence tomography to investigate the effects of PCSK9-antibodies vascular structure: Firstly, a retrospective study reported that treatment with evolocumab for 12 weeks increased fibrous-cap thickness and reduced the lipid-rich plaque proportion in 18 patients (intervention group) with acute coronary syndromes [9]. Secondly, a prospective randomized trial reported that treatment with alirocumab for 36 weeks also increased fibrous cap thickness and reduced the lipid content of plaques in 12 patients (intervention group) with coronary artery disease [10]. Most recently, the HUYGENS phase 3 trial investigated the effects of 52 weeks evolocumab treatment on fibrous cap thickness by optical coherence tomography. This double-blind study enrolled 161 patients with non-ST-elevation myocardial infarction who were randomized to statin treatment plus evolocumab versus statin treatment alone. Fibrous cap thickness significantly increased in the statin plus evolocumab group versus the statin treatment group [25]. The well-renowned GLAGOV trial demonstrated that 1 year of treatment with evolocumab reduces atheroma volume of the coronary arteries using intravascular ultrasound, another invasive method. This multi-center, randomized, and placebo-controlled study included 423 participants with coronary artery disease per group [7]. However, changes in coronary plaque composition were not detectable using virtual histology [26]. In agreement with the GLAGOV trial, the randomized, prospective ODYSSEY J-IVUS trial with 206 participants with acute coronary syndromes per group reported a nominal reduction of the total atheroma volume in response to 36 weeks of alirocumab treatment [8].

In addition to the completely novel magnetic resonance method, established ultrasoundbased methods, namely carotid intima-media thickness and flow-mediated dilatation, were used in this study. Of relevance, intima-media thickness at baseline ranged from 0.42 to 0.77 $\mathrm{mm}$ (mean 0.58) and would not meet current recommendations for significant cardiovascular disease recently defined as $\geq 1.5 \mathrm{~mm}$ by the American Society of Echocardiography [11]. This observation also highlights the importance of sophisticated cardiovascular imaging in primary prevention patients with hypercholesterolemia [27]. Accordingly, the current guidelines for the treatment of dyslipidemia endorsed by the European Atherosclerosis Society and the European Society of Cardiology consider carotid intima-media thickness as inferior to the coronary artery calcium score or the detection of significant $(>50 \%)$ stenoses (coronary angiography, computer tomography or carotid ultrasound) for very-high risk categorization [18]. Not surprisingly, carotid intima-media thickness also did not change in response to alirocumab due to the short treatment period, low sample size, and relatively low baseline intima-media thickness. Over a longer period of 12 months, a recent retrospective observational analysis suggested a reduction of carotid intima-media thickness by evolocumab in 229 patients with statin pre-treatment [28]. Moreover, carotid intima-media thickness was reduced by treatment with PCSK9-antibodies in addition to ongoing lipoprotein apheresis in only 14 heterozygous familial hypercholesterolemia patients. However, the participants of this study had higher mean baseline LDL-cholesterol of $197 \mathrm{mg} / \mathrm{dL}$ compared with the ALIROCKS study, markedly elevated lipoprotein(a) of $380 \mathrm{nmol} / \mathrm{L}$, and a much longer treatment duration of 42 months versus 10 weeks in the ALIROCKS study.

The ALIROCKS trial showed a nominal amelioration $(+41 \%)$ but no significant change of flow-dependent dilatation of the brachial artery in response to alirocumab. In contrast, 8 weeks of treatment with evolocumab increased flow-dependent dilatation in a small Italian cohort of 14 patients. Interestingly, the effect size was similar in the ALIROCKS trial but baseline values in the ALIROCKS study were lower compared with the Italian study. This difference in baseline flow-dependent dilatation values may be due to differences in patient characteristics and/or observer bias between the present study and the Italian study. All participants of the Italian study had a prior myocardial infarction and did not reach LDL-cholesterol goals despite a combination therapy of a high-dose statin and ezetimibe. 
Another recent study with 25 patients suffering from familial hypercholesterolemia (mean baseline LDL-cholesterol: $201 \mathrm{mg} / \mathrm{dL}$, mean baseline Lp(a): $69 \mathrm{mg} / \mathrm{dL}$ ) also showed amelioration of endothelial function in response to 12 weeks of treatment with PCSK9antibodies. The reason for the difference in the results may be because all ALIROCKS participants had an advanced atherosclerotic cardiovascular disease but considerably lower baseline LDL cholesterol. Of note, the characteristics of the ALIROCKS participants were consistent with recent real-world evidence from Austria [6]. Finally, differences in the results compared with previous studies may also be due to methodological limitations of the flow-mediated dilatation method [29].

Another objective of the trial was to test the effects of treatment with alirocumab on inflammatory and vascular biomarkers. Whereas a pro-inflammatory role of PCSK9 in atherosclerosis has been suggested, clinical studies did not show a significant reduction of systemic inflammatory biomarkers by PCSK9-antibodies, even in myocardial infarction [30,31]. Consistent with previous analyses of interventional PCSK9-antibody trials we detected no changes for CRP and MCP-1 and only a modest reduction of IL- 6 plasma levels [30]. In this context, it is of relevance to consider that the participants did not have elevated biomarkers of inflammation at inclusion into the study. On the other hand, arterial wall inflammation measured with positron emission tomography/computed tomography was attenuated by alirocumab without changes in circulating inflammatory markers [32]. While in-vitro investigations of the PCSK9-antibody evolocumab suggested a stimulation of angiogenesis by increased VEGF release, we could not detect changes of plasma VEGF by 10 weeks of alirocumab treatment in our clinical trial [33]. In agreement with an Italian study, we did also not detect changes of plasma P-selectin in response to short-term PCSK9antibody treatment [34]. However, another measurement time point of this Italian trial after a longer treatment (12 months) showed a significant reduction of soluble plasma P-selectin, which may suggest a time-dependent treatment effect on this vascular biomarker [34].

It is a strength of the ALIROCKS study that a novel and innovative magnetic resonancebased method to visualize and evaluate the vascular structure of the carotid arteries was used. We also included high-quality ultrasound-based vascular imaging data acquired by a single well-trained technician. Moreover, the magnetic resonance and ultrasound data were monitored by an independent clinical research organization. Finally, we were able to provide "real world" data of patients with an indication for treatment with PCSK9-antibodies. We recruited patients with diverse vascular complications, concomitant disorders, lipidlowering premedication, and lipid levels. This approach reduces the risk of selection bias but at the same time leads to a more heterogeneous collective with differences in medication as well as in locations and extent of atherosclerotic lesions. Such differences in baseline characteristics may also have an impact on the results of the ALIROCKS study.

It is a limitation of the ALIROCKS study that no control group is available. However, all participants had a medical indication for treatment with PCSK9-antibodies. Hence, it would have been unethical to withhold alirocumab from half of the participants. The magnetic resonance imaging method has not yet been implemented in patients with atherosclerosis and comparisons with healthy individuals have not been made. Therefore, reference values for fractional anisotropy values in atherosclerotic vessels versus those vessels without atherosclerosis are currently not available. The relatively low sample size may also be regarded as a weak point of the ALIROCKS study. Nevertheless, beneficial effects of short-term lipid-lowering therapy on vascular function have previously been documented in studies with similar or smaller sample sizes and with even shorter treatment duration [19-24]. On the other hand, the duration of the intervention was too short to expect significant effects on fractional anisotropy of the carotid artery and carotid intima-media thickness. However, the aim was to gain and present clinical experience and longitudinal data with the novel magnetic resonance imaging method.

To conclude, endothelial function measured with flow-dependent dilatation of the brachial artery did not significantly change in response to short-term treatment with 
alirocumab. More work would be needed to evaluate whether fractional anisotropy may be useful in clinical atherosclerosis research.

Supplementary Materials: The following are available online at https:/ / www.mdpi.com/article / 10.3390/biomedicines10010152/s1. Figure S1. Illustration of Fractional Anisotropy Evaluation by Magnetic Resonance Imaging; Figure S2. Plasma LDL-cholesterol Levels at Baseline and after 10 weeks of Alirocumab Treatment; Figure S3. Correlation of Alirocumab Treatment Effect on Flow-dependent Dilatation of the Arte-ria Brachialis and LDL-Cholesterol Lowering; Table S1. Characteristics of Trial Participants according to defined Study Populations.

Author Contributions: T.M. and G.S. designed the trial and wrote the study protocol, were responsible for regulatory authority and ethics committee approvals, documentation, statistical analysis, interpretation of data, and medical writing. D.R.L., A.B., K.M., H.S. (Harald Sourij), and H.T. were responsible for patient recruitment and patient anamnesis. G.D. conducted ultrasound-based endothelial function assessments. P.O., F.G., and G.R. were responsible for nuclear magnetic resonance imaging. T.S. and H.S. (Hubert Scharnagl) performed laboratory measurements. M.B., H.D., W.M., and U.L. critically reviewed and edited the manuscript. All authors gave scientific advice within their area of expertise and were involved in the review process of this work. All authors have read and agreed to the published version of the manuscript.

Funding: This research was funded by the Medical University of Graz and by unrestricted grants from Numares AG and Sanofi-Aventis.

Institutional Review Board Statement: The study was conducted in accordance with the Declaration of Helsinki and approved by the Ethics Committee of the Medical University of Graz (protocol code: 29-519 ex 16/17 and date of approval: 6 April 2018).

Informed Consent Statement: Informed consent was obtained from all subjects involved in the study.

Data Availability Statement: The data presented in this study are available on specific request from the corresponding author.

Acknowledgments: With thanks to the team of Numares AG for conducting the nuclear magnetic resonance spectroscopy, beyond their financial support of the trial. Special thanks to the Doctoral School of the Medical University of Graz for funding laboratory assays.

Conflicts of Interest: The costs of this study were in part defrayed using research grants from Numares AG and Sanofi-Aventis. Both commercial entities were not involved in the study design, collection, analysis, interpretation of data, the writing of this article, or the decision to submit it for publication. T. Metzner is an employee of Sanofi-Aventis and performed the scientific work for this study in his role as a doctoral student at the Medical University of Graz outside his work at Sanofi-Aventis. D.R. Leitner received personal fees from Sanofi-Aventis. H. Sourij has received research grants from Sanofi-Aventis, and personal fees from Amgen as well as Sanofi-Aventis as advisor and speaker. W. März reports grants and personal fees from Amgen, Sanofi-Aventis, and from Numares AG. H. Scharnagl has received grants and/or personal fees from Sanofi-Aventis, Amgen, and Numares AG. H. Toplak reports grants and personal fees from Sanofi-Aventis as advisor and speaker. G. Silbernagel reports grants and personal fees from Sanofi-Aventis, grants, personal fees and non-financial support from Amgen, and grants from Numares AG. All authors declare no other competing interest.

\begin{tabular}{|c|c|}
\hline Apo & Apolipoprotein \\
\hline C-IMT & Carotid-Intima-media Thickness \\
\hline COVID-19 & Coronavirus Disease-2019 \\
\hline CRP & C-Reactive Protein \\
\hline FDD & Flow-Dependent Dilatation \\
\hline HDL & High-Density Lipoprotein \\
\hline IL-6 & Interleukin- 6 \\
\hline $\operatorname{Lp}(\mathrm{a})$ & Lipoprotein-a particles \\
\hline LDL & Low-Density Lipoprotein \\
\hline MCP-1 & Monocyte Chemoattractant Protein-1 \\
\hline MRI & Magnetic Resonance Imaging \\
\hline PCSK9 & Proprotein Convertase Subtilisin/Kexin type 9 \\
\hline VEGF & Vascular Endothelial Growth Factor \\
\hline
\end{tabular}




\section{References}

1. Virani, S.S.; Catellier, D.J.; Pompeii, L.A.; Nambi, V.; Hoogeveen, R.C.; Wasserman, B.A.; Coresh, J.; Mosley, T.H.; Otvos, J.D.; Sharrett, A.R.; et al. Relation of cholesterol and lipoprotein parameters with carotid artery plaque characteristics: The Atherosclerosis Risk in Communities (ARIC) carotid MRI study. Atherosclerosis 2011, 219, 596-602. [CrossRef]

2. Tsujita, K.; Sugiyama, S.; Sumida, H.; Shimomura, H.; Yamashita, T.; Yamanaga, K.; Komura, N.; Sakamoto, K.; Oka, H.; Nakao, K.; et al. Impact of Dual Lipid-Lowering Strategy with Ezetimibe and Atorvastatin on Coronary Plaque Regression in Patients with Percutaneous Coronary Intervention: The Multicenter Randomized Controlled PRECISE-IVUS Trial. J. Am. Coll. Cardiol. 2015, 66, 495-507. [CrossRef]

3. Räber, L.; Taniwaki, M.; Zaugg, S.; Kelbæk, H.; Roffi, M.; Holmvang, L.; Noble, S.; Pedrazzini, G.; Moschovitis, A.; Lüscher, T.F.; et al. Effect of high-intensity statin therapy on atherosclerosis in non-infarct-related coronary arteries (IBIS-4): A serial intravascular ultrasonography study. Eur. Heart J. 2015, 36, 490-500. [CrossRef]

4. Schwartz, G.G.; Steg, P.G.; Szarek, M.; Bhatt, D.L.; Bittner, V.A.; Diaz, R.; Edelberg, J.M.; Goodman, S.G.; Hanotin, C.; Harrington, R.A.; et al. Alirocumab and Cardiovascular Outcomes after Acute Coronary Syndrome. N. Engl. J. Med. 2018, 379, $2097-2107$. [CrossRef]

5. Sabatine, M.S.; Giugliano, R.P.; Keech, A.C.; Honarpour, N.; Wiviott, S.D.; Murphy, S.A.; Kuder, J.F.; Wang, H.; Liu, T.; Wasserman, S.M.; et al. Evolocumab and Clinical Outcomes in Patients with Cardiovascular Disease. N. Engl. J. Med. 2017, 376, 1713-1722. [CrossRef]

6. $\quad$ Leitner, D.R.; Toplak, H.; Kedenko, L.; Steinmaurer, T.; Gräff, V.; Metzner, T.; Schwaiger, E.M.; Prager, R. Efficacy and tolerability of alirocumab in Austrian clinical practice-results of the non-interventional PEARL-AT study. Curr. Med. Res. Opin. 2020, 36, 1419-1425. [CrossRef]

7. Nicholls, S.J.; Puri, R.; Anderson, T.; Ballantyne, C.M.; Cho, L.; Kastelein, J.J.; Koenig, W.; Somaratne, R.; Kassahun, H.; Yang, J.; et al. Effect of Evolocumab on Progression of Coronary Disease in Statin-Treated Patients: The GLAGOV Randomized Clinical Trial. JAMA 2016, 316, 2373-2384. [CrossRef] [PubMed]

8. Ako, J.; Hibi, K.; Tsujita, K.; Hiro, T.; Morino, Y.; Kozuma, K.; Shinke, T.; Otake, H.; Uno, K.; Louie, M.J.; et al. Effect of Alirocumab on Coronary Atheroma Volume in Japanese Patients with Acute Coronary Syndrome-The ODYSSEY J-IVUS Trial. Circ. J. 2019, 83, 2025-2033. [CrossRef] [PubMed]

9. Yano, H.; Horinaka, S.; Ishimitsu, T. Effect of evolocumab therapy on coronary fibrous cap thickness assessed by optical coherence tomography in patients with acute coronary syndrome. J. Cardiol. 2020, 75, 289-295. [CrossRef] [PubMed]

10. Sugizaki, Y.; Otake, H.; Kawamori, H.; Toba, T.; Nagano, Y.; Tsukiyama, Y.; Yanaka, K.; Yamamoto, H.; Nagasawa, A.; Onishi, H.; et al. Adding Alirocumab to Rosuvastatin Helps Reduce the Vulnerability of Thin-Cap Fibroatheroma: An ALTAIR Trial Report. JACC Cardiovasc. Imaging 2020, 13, 1452-1454. [CrossRef]

11. Johri, A.M.; Nambi, V.; Naqvi, T.Z.; Feinstein, S.B.; Kim, E.S.; Park, M.M.; Becher, H.; Sillesen, H. Recommendations for the Assessment of Carotid Arterial Plaque by Ultrasound for the Characterization of Atherosclerosis and Evaluation of Cardiovascular Risk: From the American Society of Echocardiography. J. Am. Soc. Echocardiogr. 2020, 33, 917-933. [CrossRef] [PubMed]

12. Bots, M.L.; Evans, G.W.; Tegeler, C.H.; Meijer, R. Carotid Intima-media Thickness Measurements: Relations with Atherosclerosis, Risk of Cardiovascular Disease and Application in Randomized Controlled Trials. Chin. Med. J. 2016, 129, 215-226. [CrossRef]

13. Hafner, F.; Kieninger, A.; Meinitzer, A.; Gary, T.; Froehlich, H.; Haas, E.; Hackl, G.; Eller, P.; Brodmann, M.; Seinost, G. Endothelial dysfunction and brachial intima-media thickness: Long term cardiovascular risk with claudication related to peripheral arterial disease: A prospective analysis. PLoS ONE 2014, 9, e93357. [CrossRef]

14. Hafner, F.; Seinost, G.; Gary, T.; Froehlich, H.; Pilger, E.; Brodmann, M. Are flow-mediated vasodilatation and intima-media thickness of the brachial artery associated with restenosis after endovascular treatment of peripheral arterial occlusive disease? Eur. Radiol. 2010, 20, 2533-2540. [CrossRef] [PubMed]

15. Vlachopoulos, C.; Xaplanteris, P.; Aboyans, V.; Brodmann, M.; Cífková, R.; Cosentino, F.; De Carlo, M.; Gallino, A.; Landmesser, U.; Laurent, S.; et al. The role of vascular biomarkers for primary and secondary prevention. A position paper from the European Society of Cardiology Working Group on peripheral circulation: Endorsed by the Association for Research into Arterial Structure and Physiology (ARTERY) Society. Atherosclerosis 2015, 241, 507-532.

16. Opriessnig, P.; Silbernagel, G.; Krassnig, S.; Reishofer, G. Magnetic resonance microscopy diffusion tensor imaging of collagen fibre bundles stabilizing an atherosclerotic plaque of the common carotid artery. Eur. Heart J. 2018, 39, 3337. [CrossRef]

17. Opriessnig, P.; Mangge, H.; Stollberger, R.; Deutschmann, H.; Reishofer, G. In vivo cardiovascular magnetic resonance of 2D vessel wall diffusion anisotropy in carotid arteries. J. Cardiovasc. Magn. Reson. 2016, 18, 81. [CrossRef]

18. Mach, F.; Baigent, C.; Catapano, A.L.; Koskinas, K.C.; Casula, M.; Badimon, L.; Chapman, M.J.; De Backer, G.G.; Delgado, V.; Ference, B.A.; et al. 2019 ESC/EAS Guidelines for the management of dyslipidaemias: Lipid modification to reduce cardiovascular risk. Eur. Heart J. 2020, 41, 111-188. [CrossRef]

19. Landmesser, U.; Bahlmann, F.; Mueller, M.; Spiekermann, S.; Kirchhoff, N.; Schulz, S.; Manes, C.; Fischer, D.; de Groot, K.; Fliser, D.; et al. Simvastatin versus ezetimibe: Pleiotropic and lipid-lowering effects on endothelial function in humans. Circulation 2005, 111, 2356-2363. [CrossRef]

20. Yoshida, O.; Kondo, T.; Kureishi-Bando, Y.; Sugiura, T.; Maeda, K.; Okumura, K.; Murohara, T. Pitavastatin, an HMG-CoA reductase inhibitor, ameliorates endothelial function in chronic smokers. Circ. J. 2010, 74, 195-202. [CrossRef] [PubMed] 
21. Amudha, K.; Choy, A.M.; Mustafa, M.R.; Lang, C.C. Short-term effect of atorvastatin on endothelial function in healthy offspring of parents with type 2 diabetes mellitus. Cardiovasc. Ther. 2008, 26, 253-261. [CrossRef]

22. Karatzis, E.; Lekakis, J.; Papamichael, C.; Andreadou, I.; Cimponeriu, A.; Aznaouridis, K.; Papaioannou, T.G.; Protogerou, A.; Mavrikakis, M. Rapid effect of pravastatin on endothelial function and lipid peroxidation in unstable angina. Int. J. Cardiol. 2005, 101, 65-70. [CrossRef] [PubMed]

23. Maulucci, G.; Cipriani, F.; Russo, D.; Casavecchia, G.; Di Staso, C.; Di Martino, L.F.M.; Ruggiero, A.; Di Biase, M.; Brunetti, N.D. Improved endothelial function after short-term therapy with evolocumab. J. Clin. Lipidol. 2018, 12, 669-673. [CrossRef]

24. Di Minno, A.; Gentile, M.; Iannuzzo, G.; Calcaterra, I.; Tripaldella, M.; Porro, B.; Cavalca, V.; Di Taranto, M.D.; Tremoli, E.; Fortunato, G.; et al. Endothelial function improvement in patients with familial hypercholesterolemia receiving PCSK-9 inhibitors on top of maximally tolerated lipid lowering therapy. Thromb. Res. 2020, 194, 229-236. [CrossRef] [PubMed]

25. Nicholls, S.J.; Nissen, S.E.; Prati, F.; Windecker, S.; Kataoka, Y.; Puri, R.; Hucko, T.; Kassahun, H.; Liao, J.; Somaratne, R.; et al. Assessing the impact of PCSK9 inhibition on coronary plaque phenotype with optical coherence tomography: Rationale and design of the randomized, placebo-controlled HUYGENS study. Cardiovasc. Diagn. Ther. 2021, 11, 120-129. [CrossRef]

26. Nicholls, S.J.; Puri, R.; Anderson, T.; Ballantyne, C.M.; Cho, L.; Kastelein, J.J.; Koenig, W.; Somaratne, R.; Kassahun, H.; Yang, J.; et al. Effect of Evolocumab on Coronary Plaque Composition. J. Am. Coll. Cardiol. 2018, 72, 2012-2021. [CrossRef] [PubMed]

27. Randrianarisoa, E.; Rietig, R.; Jacob, S.; Blumenstock, G.; Haering, H.-U.; Rittig, K.; Balletshofer, B.; Information, R. Normal values for intima-media thickness of the common carotid artery-an update following a novel risk factor profiling. Vasa 2015, 44, 444-450. [CrossRef]

28. Hirai, K.; Imamura, S.; Hirai, A.; Ookawara, S.; Morishita, Y. Effects of Evolocumab on Carotid Intima-Media Thickness and Clinical Parameters in Patients Taking a Statin. J. Clin. Med. 2020, 9, 2256. [CrossRef]

29. Hijmering, M.L.; Stroes, E.S.; Pasterkamp, G.; Sierevogel, M.; Banga, J.D.; Rabelink, T.J. Variability of flow mediated dilation: Consequences for clinical application. Atherosclerosis 2001, 157, 369-373. [CrossRef]

30. Momtazi-Borojeni, A.A.; Sabouri-Rad, S.; Gotto, A.M.; Pirro, M.; Banach, M.; Awan, Z.; E Barreto, G.; Sahebkar, A. PCSK9 and inflammation: A review of experimental and clinical evidence. Eur. Heart J. Cardiovasc. Pharmacother. 2019, 5, 237-245. [CrossRef]

31. Trankle, C.R.; Wohlford, G.; Buckley, L.F.; Kadariya, D.; Ravindra, K.; Markley, R.; Park, T.S.; Potere, N.; Van Tassell, B.W.; Abbate, A. Alirocumab in Acute Myocardial Infarction: Results from the Virginia Commonwealth University Alirocumab Response Trial (VCU-AlirocRT). J. Cardiovasc. Pharmacol. 2019, 74, 266-269. [CrossRef] [PubMed]

32. Hoogeveen, R.M.; Opstal, T.S.; Kaiser, Y.; Stiekema, L.C.; Kroon, J.; Knol, R.J.; Bax, W.A.; Verberne, H.J.; Cornel, J.; Stroes, E.S. PCSK9 Antibody Alirocumab Attenuates Arterial Wall Inflammation Without Changes in Circulating Inflammatory Markers. JACC Cardiovasc. Imaging 2019, 12, 2571-2573. [CrossRef] [PubMed]

33. Safaeian, L.; Vaseghi, G.; Jabari, H.; Dana, N. Evolocumab, a proprotein convertase subtilisin/kexin type 9 inhibitor, promotes angiogenesis in vitro. Can. J. Physiol. Pharmacol. 2019, 97, 352-358. [CrossRef] [PubMed]

34. Barale, C.; Bonomo, K.; Frascaroli, C.; Morotti, A.; Guerrasio, A.; Cavalot, F.; Russo, I. Platelet function and activation markers in primary hypercholesterolemia treated with anti-PCSK9 monoclonal antibody: A 12-month follow-up. Nutr. Metab. Cardiovasc. Dis. 2020, 30, 282-291. [CrossRef] [PubMed] 